\title{
Entropy generation in an inclined porous channel with suction/injection
}

https://doi.org/10.1515/nleng-2018-0116

Received August 8, 2018; revised November 2, 2018; accepted January 11, 2019.

Abstract: The present work is concerned with an analytical study of entropy generation in viscous, incompressible fluid in an inclined channel with porous walls. The solution of the governing equations were obtained in closed form. The expression for the irreversibility ratio was also obtained and the results were presented graphically and extensively discussed for different values of the dimensionless parameters. The result indicates that wall inclination enhance entropy generation.

Keywords: entropy generation, irreversibility ratio, suction/injection

\section{Introduction}

Considering first law of thermodynamics, temperature distribution of fluid and heat transfer coefficient within the channel can be obtained, this law however does not account for the relative contribution of viscosity and heat convection for entropy generation in a system [1]. Second law analysis becomes suitable since it gives information regarding the relative viscosity and heat transfer in the system. This law plays a vital role in predicting the performance of the engineering processes, since entropy generation measures the destruction of the available work of the system. In order to improve its performance, it is important to determine factors responsible for entropy generation in the system. This process is made easy since by second law analysis, many different interactions in the process can be compared to identify the major sources of energy losses. This method was introduced by Bejan [2-4] and due to the importance of minimizing the entropy generation, many

\footnotetext{
Basant K. Jha, Department of Mathematics, Ahmadu Bello University, Zaria, Nigeria

*Corresponding Author: Taiwo S. Yusuf, Department of Mathematics, Ahmadu Bello University, Zaria, Nigeria,

E-mail: taiyeee@yahoo.com
}

researchers in the last decade have developed interest in this area of study.

In particular, Makinde and Maserumule [5] investigated entropy generation in a Couette flow of viscous incompressible fluid and concluded that viscous dissipation irreversibility dominates near the moving plate while heat transfer irreversibility dominates near the stationary plate. Makinde and Gbolagade [6] studied second law analysis of incompressible viscous flow through an inclined channel with isothermal walls. Makinde and Osalusi [7] investigated entropy generation in a liquid film falling along an inclined porous heated plate. They found that heat transfer irreversibility dominates the entropy generation near the inclined heated porous plate while the fluid friction irreversibility dominates near the liquid-free surface. Ajibade and Jha [8] studied entropy generation under the effect of suction/injection and they concluded that an increase in suction on the cold porous plate increases dominance of heat transfer irreversibility, while an increase in injection on the cold porous plate decreases the dominance of heat transfer irreversibility over the fluid friction irreversibility

Hooman et al. [9] numerically studied entropy generation for forced convection in a porous channel with isoflux or isothermal walls and concluded that different arrangement of the parameter lead to completely different behaviour for both entropy generation number and Bejan number. Aziz [10] presented entropy generation in pressure gradient assisted Couette flow. Four different combinations of thermal boundary conditions are investigated. His results illustrate the effect of pressure gradient, temperature asymmetry, heat flux, convection Biot numbers, and ambient temperatures and concluded that for certain combinations of thermal variables, the total entropy generated is minimized. Using local similarity solution technique and shooting quadrature, Makinde [11] numerically investigated second law analysis for variable viscosity hydro-magnetic boundary layer flow with thermal radiation and Newtonian heating while in conjunction with Aziz [12] presented a numerical study of Second law analysis for a variable viscosity plane Poiseuille flow with asymmetric convective cooling using an efficient numerical shooting technique with a fourth order Runge-Kutta 
algorithm. Vyas and Ranjan [13] analytically examined entropy analysis for MHD generalized Couette flow in a composite duct. In another work, using entropy generation analysis, the mixed convection flow between two vertical concentric pipes with constant heat flux at the boundaries and MHD flow effects is considered by Mahian et al. [14]. It is concluded that with an increase in the Hartmann number, the energy cost increases.

Later on, thermo-economic analysis of a heat exchanger was performed by Mahian et al. [15] to estimate the total costs due to entropy generation for the flow of a refrigerant inside the heat exchanger. It was found that in the cases investigated, the parameter $\mathrm{Gr} / \mathrm{Re}$ has no considerable effect on entropy generation. The generalized axial Couette flow of an Ostwald-de Waele power law reactive fluid between concentric cylindrical pipes has been numerically investigated by Makinde [16] using the shooting method coupled with the Runge-Kutta-Fehlberg integration technique.

In this present study, special attention has been given to the combined effects of porosity and wall inclination with symmetric heating on entropy generation and irreversibility ratio. Problem is formulated, solved and discussed.

\section{Mathematical analysis}

Steady flow of viscous incompressible fluid is considered in an inclined heated channels formed by two infinite parallel porous walls. The $\mathrm{x}$-direction is taken parallel to one of the channel porous wall while the $y$ - direction is taken normal to it.

The geometry of the system under consideration in this present study is shown schematically in Fig. 1 consisting of fluid flowing steadily downstream in the $x$ - direction. The basic equations governing the flow of viscous incompressible fluid between two heated inclined parallel plates are;

Conservation of mass

$$
\nabla \cdot \vec{V}=0
$$

Conservation of momentum

$$
\frac{\partial \vec{V}}{\partial t}+(\vec{V} \cdot \nabla) \vec{V}=\nu \nabla^{2} \vec{V}-\frac{1}{\rho} \nabla P+\vec{g}
$$

and Conservation of energy

$$
\frac{\partial T}{\partial t}+(\vec{V} \cdot \nabla) T=\frac{k}{\rho C_{P}} \nabla^{2} T
$$

Where $\nabla=\frac{\partial}{\partial x} \underline{i}+\frac{\partial}{\partial y} \underline{j}+\frac{\partial}{\partial z} \underline{k}$. The physical quantities $\rho, v, \rho_{0}, C_{P}, \vec{V}, T$ and $\mathrm{k}$ are defined in nomenclature.

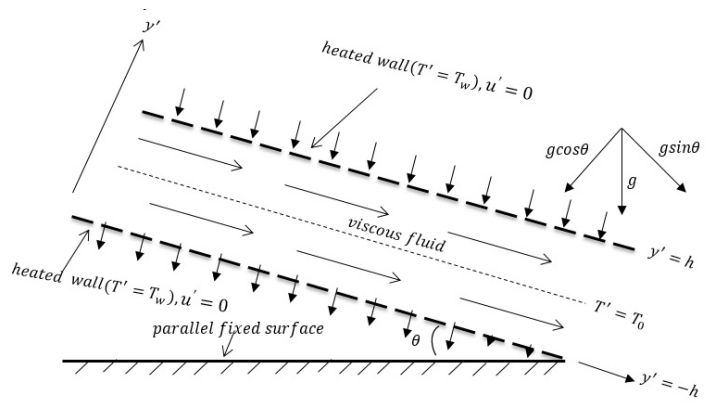

Fig. 1: Schematic diagram

We consider a two dimensional flow so that $\vec{V}=$ $(u, v, 0)$, where $u$ and $v$ are the horizontal and vertical components of velocity, respectively. Moreover, it is assumed that the flow is along $\mathrm{x}$-axis which is steady fully developed and depending on y alone (Fig. 1) so that Eq. (1) becomes

$$
\frac{\partial v}{\partial y}=0
$$

Integrating Eq. (4), we have the vertical velocity as $v=-v_{0}$ (a constant) which is the velocity of suction/injection.

The dimensional governing momentum and energy equations for the steady flow of viscous incompressible fluid with the associated boundary conditions are given by

$$
\begin{aligned}
v \frac{d^{2} u^{\prime}}{d y^{\prime 2}}+g \sin \theta & =-v_{0} \frac{d u^{\prime}}{d y^{\prime}} \\
u^{\prime} \frac{\partial T^{\prime}}{\partial x^{\prime}}-v_{0} \frac{\partial T^{\prime}}{\partial y^{\prime}} & =\alpha \frac{\partial^{2} T^{\prime}}{\partial y^{\prime 2}} \\
u^{\prime}( \pm h) & =0 \\
T^{\prime}\left(0, y^{\prime}\right) & =T_{0} \\
T^{\prime}\left(x^{\prime}, \pm h\right) & =T_{w}
\end{aligned}
$$

The non-dimensional quantities in the above equations are defined as

$$
\begin{aligned}
& y=\frac{y^{\prime}}{h}, s=\frac{v_{0} h}{v}, \\
& U=\frac{u^{\prime} v}{\left[g h^{2} \sin \theta\right]}, \\
& T=\frac{T^{\prime}-T_{0}}{T_{w}-T_{0}}, \\
& \operatorname{Pr}=\frac{v}{\alpha}, \\
& x=\frac{x^{\prime} \alpha v}{\left[g h^{4} \sin \theta\right]}
\end{aligned}
$$


In dimensionless form, Eq. (6) to (9) are obtained as

$$
\begin{gathered}
\frac{d^{2} U}{d y^{2}}+s \frac{d U}{d y}=-1 \\
U \frac{d T}{d x}=\frac{d^{2} T}{d y^{2}}+s P r \frac{d T}{d y} \\
U( \pm 1)=0 \\
T(0, y)=0 \\
T(x, \pm 1)=1
\end{gathered}
$$

Where $\operatorname{Pr}$ is the Prandtl number which is inversely proportional to the thermal diffusivity of the working fluid, $s$ is the dimensionless suction/injection parameter. Positive values of $s$ denotes injection at the porous wall $y^{\prime}=h$ with a corresponding suction on the wall $y^{\prime}=-h$ while negative values denote injection at the porous plate $y^{\prime}=-h$ with a corresponding suction on the other plate. The physical quantities used in Eq. (10) are defined in the nomenclature.

The solution to Eq. (11) and (12) with the boundary conditions (13) to (15) is given by

$$
\begin{gathered}
U(y)=\frac{1}{s}\left[\frac{\cosh (s)-e^{-s y}}{\sinh ?(s)}\right]-\frac{y}{s} \\
T(x, y)=A x
\end{gathered}
$$

Where

$$
\begin{aligned}
& A= \\
& {\left[\frac{1}{x-\frac{k_{3}}{S P r}-k_{2}{ }^{*} e^{-s P r}+\frac{1}{2 s} \frac{\left(2-2 s y P r+(y s P r)^{2}\right)}{(s P r)^{3}}-\frac{e^{-s y}}{B s^{2}(P r-1)}-\frac{C(y s P r-1)}{B(s P r)^{2}}}\right]} \\
& k_{3}=\frac{C}{B s P r}-\frac{\operatorname{Prcosh}(s)}{B s(\operatorname{Pr}-1)}-k_{2}{ }^{*} \operatorname{sPr} \cosh (s P r)+\left[\frac{2+(s P r)^{2}}{2 s(s P r)^{2}}\right] \\
& K_{2}{ }^{*}=\frac{1}{2 \sinh ?(s P r)}\left[\frac{2 C}{B s P r}+\frac{2}{s(s P r)^{2}}\right]-\frac{1}{B s^{2}(P r-1)} \frac{\sinh (s)}{\sinh (s P r)} \\
& B=s \times \sinh (s) \\
& C=\cosh (s)
\end{aligned}
$$

\section{Entropy generation rate}

When we consider the flow of a Newtonian incompressible fluid under the influence of Fourier law of heat conduction, the Cartesian coordinates of the volumetric rate of entropy generation is given by Bejan [9] as

$$
\begin{aligned}
E_{G}= & \frac{k}{T_{0}{ }^{2}}\left(\left(\frac{\partial T}{\partial x}\right)^{2}+\left(\frac{\partial T}{\partial y}\right)^{2}\right) \\
& +\frac{\mu}{T_{0}}\left(2\left\{\left(\frac{\partial u}{\partial x}\right)^{2}+\left(\frac{\partial v}{\partial y}\right)^{2}\right\}+\left[\frac{\partial u}{\partial y}+\frac{\partial v}{\partial x}\right]^{2}\right)
\end{aligned}
$$

The above form of entropy generation shows that the irreversibility is due to two effects; conduction $(k)$ and viscosity $(\mu)$. As long as one of temperature or velocity gradient is present in a medium, entropy generation rate is finite and positive.

In many fundamental convective heat transfer problem, Velocity and temperature distributions are simplified assuming that the flow is hydro-dynamically developed $\left(\frac{\partial \bar{V}}{\partial x}\right)=0$ and thermally developing $\left(\frac{\partial T}{\partial x} \neq 0\right)$ or thermally developed $\left(\frac{\partial T}{\partial x}=0\right)$ [20] Eq. is reduced to the following form:

$$
E_{G}=\frac{k}{T_{0}^{2}}\left(\left(\frac{\partial T}{\partial x}\right)^{2}+\left(\frac{\partial T}{\partial y}\right)^{2}\right)+\frac{\mu}{T_{0}}\left(\frac{\partial u}{\partial y}\right)^{2}
$$

In dimensionless form, $E_{G}$ is given as the entropy generation number $N_{S}$ which is, by definition, equal to the ratio of the actual entropy generation rate to the characteristics entropy transfer rate $\left(E_{G, C}\right)$ the entropy transfer rate is given by White [18] as:

$$
E_{G, C}=\left(\frac{q^{2}}{k T_{0}^{2}}\right)=\left(\frac{k(\Delta T)^{2}}{L^{2} T_{0}^{2}}\right)
$$

Where $T_{0}$ is the absolute reference temperature, $q$ is the heat flux, $\Delta T$ is the temperature difference and $\mathrm{L}$ is the characteristics length depending on the geometry of the channel and problem type. The expression at the center of the Eq. (20) is used for isoflux boundary condition while that on the right hand side is used for isothermal boundary condition.

In terms of the dimensionless velocity and temperature, the entropy generation number becomes

$$
N_{s}=\frac{1}{P e^{2}}\left(\frac{\partial T}{\partial x}\right)^{2}+\left(\frac{\partial T}{\partial y}\right)^{2}+\frac{B r}{\Omega}\left(\frac{\partial U}{\partial y}\right)^{2}=N_{x}+N_{y}+N_{f}
$$

Where

$$
N_{x}=\frac{1}{p e^{2}}\left\{A-\frac{-\chi}{F^{2}}\right\}^{2}
$$

$$
\begin{aligned}
F= & {\left[x-\frac{k_{3}}{s \cdot P r}-k_{2}{ }^{\star} e^{-s P r}+\frac{1}{2 s} \frac{\left(2-2 s y P r+(y s P r)^{2}\right)}{(s P r)^{3}}\right.} \\
& \left.-\frac{e^{-s y}}{B s^{2}(P r-1)}-\frac{C(y s P r-1)}{B(s P r)^{2}}\right]
\end{aligned}
$$




$$
\begin{gathered}
N_{y}=\frac{x^{2}\left[k_{2}{ }^{*} s P r e^{-s P r y}+\frac{(y s P r-1)}{s(s P r)^{2}}+\frac{e^{-s y}}{B s(P r-1)}-\frac{C}{B s P r}\right]^{2}}{\left[\chi-\frac{k_{3}}{s \cdot P r}-k_{2}{ }^{*} e^{-s P r}+\frac{1}{2 s} \frac{\left(2-2 s y P r+(y s P r)^{2}\right)}{(s P r)^{3}}-\frac{e^{-s y}}{B s^{2}(P r-1)}-\frac{C(y s P r-1)}{B(s P r)^{2}}\right]^{4}} \\
N_{f}=\frac{B r}{\Omega}\left[\frac{e^{-s y}}{\sinh (s)}-\frac{1}{s}\right]^{2}
\end{gathered}
$$

The dimensionless parameters $P e$ $=$ $\left(\mu g h^{3} \sin \theta\right) / \alpha \mu, B r=\left(\rho^{2} g^{2} h^{4} \sin ^{2} \theta\right) / \mu k\left(T_{w}-T_{0}\right)$ and $\Omega=\left(T_{w}-T_{0}\right) / T_{0}$ are the peclet number, Brinkman number and temperature difference parameter respectively, the first term $N_{x}$ represents the entropy generation by heat transfer due to axial conduction, the second term $N_{y}$ denotes the entropy generation due to heat transfer across different fluid sections(transverse) within the channel and the last term is $N_{f}$ is the entropy generation due to fluid friction.

\section{Irreversibility analysis}

Expression for entropy generation Eq. (21) gives the spatial distribution of entropy but fails to identify the relative contributions of each irreversibility to the total entropy generation. In order to have an idea whether it is the fluid friction or heat transfer irreversibility that dominates the total entropy, Bejan [19] defined the irreversibility distribution ratio $(\phi)$ as the ratio of the entropy generation due to viscous dissipation $\left(N_{f}\right)$ to heat transfer $\left(N_{x}+N_{y}\right)$. That is,

$$
\phi=\frac{N_{f}}{N_{x}+N_{y}}
$$

For $0 \leq \phi<1$, implies that heat transfer entropy generation dominates the irreversibility ratio while $\phi>1$, indicates that entropy generation due to fluid friction dominates the flow and $\phi=1$, signifies that heat transfer and fluid friction have equal contribution of entropy generation to irreversibility ratio.

In many engineering designs and energy optimization problem, the contribution of heat transfer entropy generation to the total entropy generation rate is required. Hence, Paoletti et al. [20] presented an alternative irreversibility distribution parameter in terms of Bejan number (Be) and defined it as the ratio of the entropy generation due to heat transfer $\left(N_{x}+N_{y}\right)$ to the total entropy generation $\left(N_{S}\right)$. The Bejan number is defined mathematically as

$$
B e=\frac{N_{x}+N_{y}}{N_{s}}=\frac{1}{1+\varnothing}
$$

Obviously, the Bejan number ranges from 0 to $1 . B e=$ 0 , is the limit where the irreversibility is dominated by fluid friction effect while $B e=1$, shows that entropy is generated solely by heat transfer irreversibility. The contribution of heat transfer and fluid friction to entropy generation is equal when $B e=0.5$. According to Bejan [21], the viscous irreversibility term in eq. (21) may not be negligible, implying that it may be considered in entropy generation analysis, even in cases when the viscous dissipation term has been neglected in the energy equation which is the case in the present problem. Similar analysis has been presented in the work of Hooman and Gurgenci [22]. In many heat transfer problems, it is often possible and convenient to neglect the viscous dissipation term in the energy equation. This is particularly the case in heat transfer through gases at subsonic velocities (Bejan [23])

\section{Result and discussion}

The role of suction/injection on entropy generation in flow of viscous incompressible fluid in an inclined porous channel is presented. The dimensionless parameters governing the flow are Prandtl number $(\mathrm{Pr})$, which is inversely proportional to the thermal diffusivity of the working fluid, Brinkman number $(\mathrm{Br})$, Peclet number $(\mathrm{Pe})$ and the suction/injection parameter $(s)$. For numerical validation of our analysis, the values of $\mathrm{Pr}$ are chosen between the nondimensional values of 0.001 and 7.0 to accommodate fluids like mercury (0.008-0.041), water vapor (0.882-0.994), oxygen (0.729-0.759), air (0.703-0.784), water (5.18-8.91), the value of the suction/injection parameter $s$ is taken between -4 to 4 , to represent both injection as well as suction etc. (Lienhard and Lienhard [24]). The numerical calculation for the dimensionless velocity $(U)$, temperature $(T)$, irreversibility ratio $(\phi)$ and entropy generation $(N s)$ have been carried out with the aid of MATLAB and the effect of the dimensionless parameter on them are shown graphically in Figs. 2-11.

Fig. 2 shows the variation of velocity for different values of suction/injection parameter. It can be observed that the fluid velocity is parabolic in nature for different values of $s$. Considering $s>0$, velocity decreases with increase in injection on the inclined heated wall $s(y=1)$, while an increase in suction on the wall $y=-1$ has no significant effect on the velocity. However, velocity is seen to decrease as we approach the heated wall. Fig. 3 and 4 shows the temperature variation for different values of $s$ and $P r$ respectively. In Fig. 3, fluid temperature is observed to increase towards the wall with maximum temperature on the heated walls. Fig. 4 revealed that fluid temperature increases as $P r$ increases. In reality, as $P r$ increases, 
the thermal diffusivity of the fluid decreases leading to decrease in the diffusion of the heat generated by fluid friction within the channel. As a result, we have accumulation of heat within the channel leading to increase in fluid temperature.

The ratio of entropy generation due to fluid viscous dissipation to entropy generation due to heat transfer is presented in Figs. 5-9. Fig. 5, 6 and 7 shows the graph of irreversibility ratio for different values of group parameter $\left(\mathrm{Br} \Omega^{-1}\right)$, axial distance $(x)$ and $(P e)$ respectively. The group parameter is an important parameter which helps in the analysis of the irreversibility, since it shows the ratio of the effect of viscosity to temperature difference in the system. We observed from these figures that irreversibility ratio increases from the middle of the inclined channel to its maximum values, then decrease towards the heated channel walls with entropy generation due to heat transfer dominating for all values of $x$ and $P e$ (Fig. 6,7). However, entropy generation due to fluid friction dominates the irreversibility ratio for values $\mathrm{Br} \Omega^{-1} \geq$ 1 , in the interval $-0.8 \leq y \leq-0.2$ and $0.8 \leq y \leq 0.2$ (Fig. 5).

The influence of different values of Prandtl number $(\mathrm{Pr})$ on the irreversibility ratio is shown in Fig. 8. The irreversibility ratio is observed to increase transversely from the centerline towards the heated wall $y=1$, with a decrease in $P r$, a change in trend is observed close to the wall as irreversibility ratio increase with $\operatorname{Pr}$ for all $\operatorname{Pr} \geq 4$. A critical look into the graph shows that the irreversibility ratio increases from the centerline of the channel with $\operatorname{Pr}$ toward the wall $y=-1$, till it reaches its maximum and then eventually decreases towards the heated wall $y=-1$. It is interesting to note that entropy generation due to heat transfer greatly dominates the fluid friction in the irreversibility for different values of $P r$. The irreversibility ratio for different values of suction/injection parameter $s$ is presented in Fig. 9. We observed two region of maximum value of $\varnothing$, for all values of $s$ and a minimum point close to the centerline of the channel. It will be interesting to note that heat transfer irreversibility dominates throughout the channel. Its dominance can however be further enhanced by increasing injection close to the heated wall $y=1$.

Entropy generation for different dimensionless parameter is presented in Fig. 10 and 11. Fig. 10 shows the entropy generation for different values of group parameter $\left(B r \Omega^{-1}\right)$. We observed that entropy generation increases on both walls with increase in $\left(B r \Omega^{-1}\right)$, while the minimum value of entropy is found within the channel $(y=0)$. i.e., available energy in transverse direction at the middle of the channel is maximum. As expected, entropy generation on the wall with suction $(y=-1)$ is higher when compared to the entropy on the wall with injection $(y=1)$.
This trend is however reversed by injecting through the wall $(y=-1)$ and sucking the fluid from the wall $(y=1)$. Variation of entropy generation with suction/injection parameter is presented in Fig. 11. The graph shows that increase in suction on the wall $(y=-1)$ increases entropy generation while increase in injection on the wall $(y=1)$ decreases the entropy, indicating that introduction of suction/injection parameter encourages entropy generation in the inclined channel.

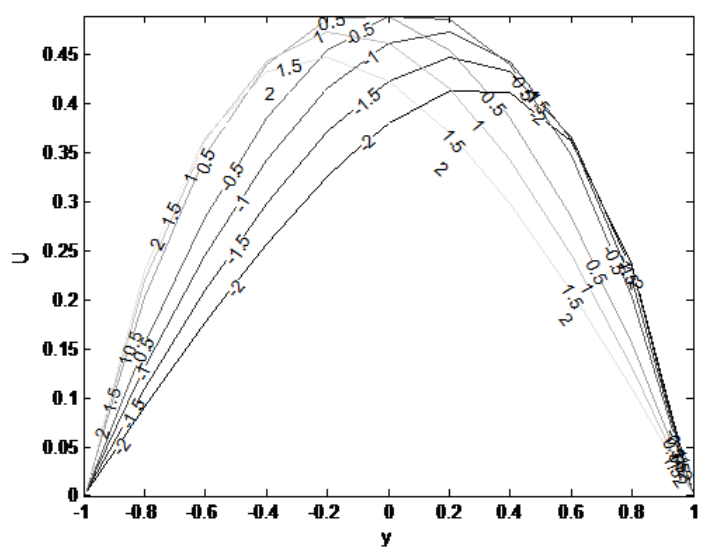

Fig. 2: velocity profile for different values of s

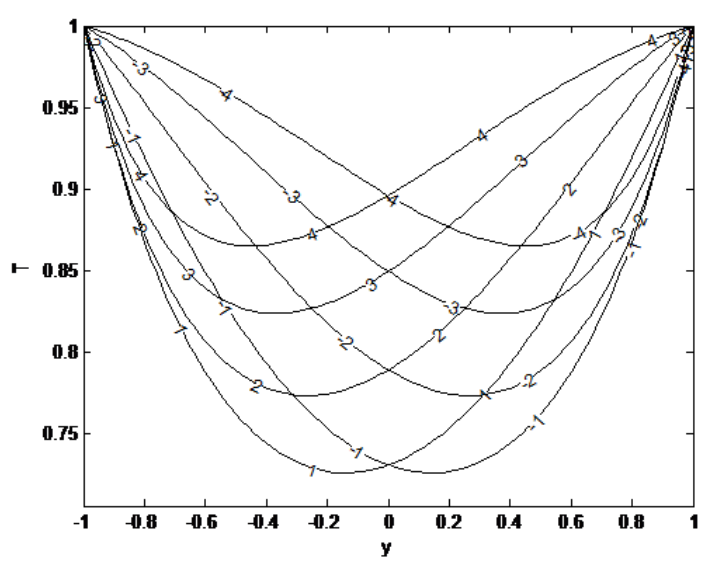

Fig. 3: Temperature profile for different s $(\operatorname{Pr}=0.71)$

\section{Conclusion}

This paper presents the study of entropy generation in an inclined channel with porous walls. The velocity and tem- 


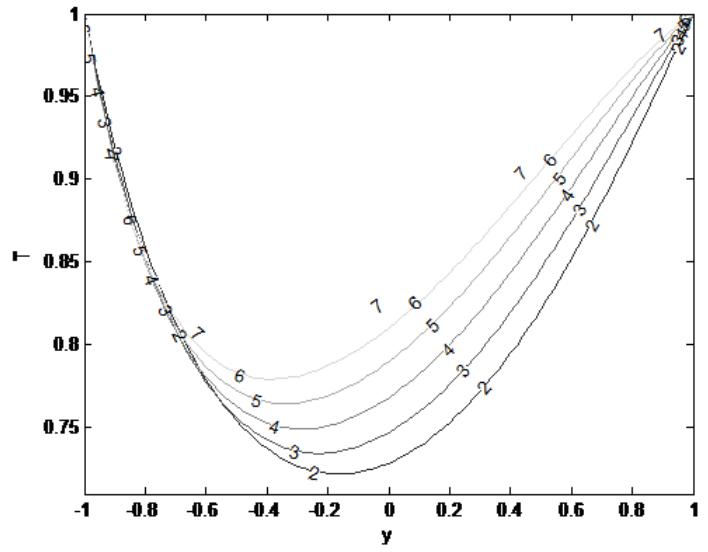

Fig. 4: Temperature profile for different values of $\operatorname{Pr}(s=0.5)$

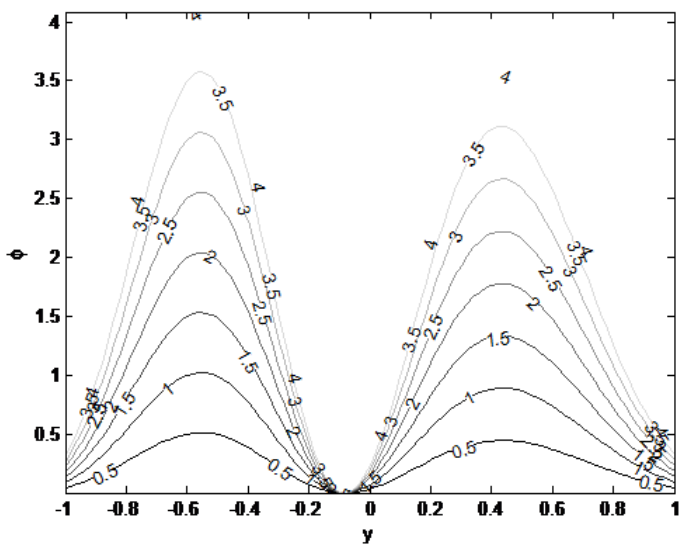

Fig. 5: Irreversibility ratio for different values of $\mathrm{Br}^{-1}(s=0.5, x=$ $0.1, \operatorname{Pr}=0.71, P e=7.1$ )

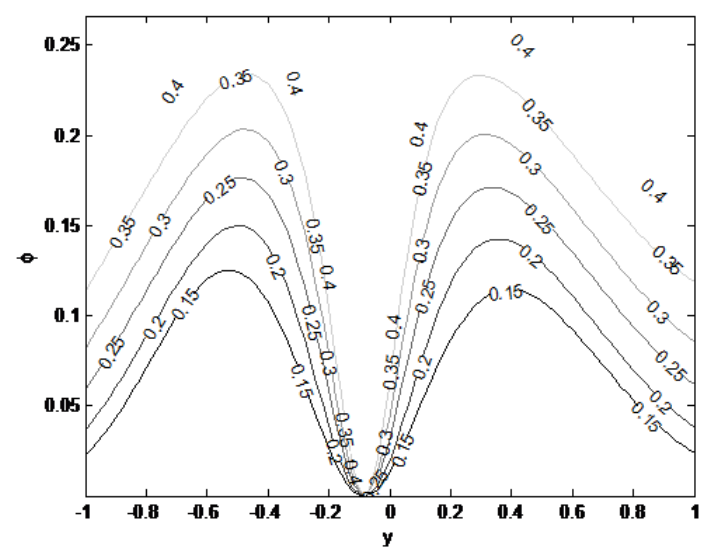

Fig. 6: Irreversibility ratio for different values of $\mathrm{x}(s=0.5, \operatorname{Pr}=$ $0.71, \operatorname{Pe}=7.1, \mathrm{Br} \Omega^{-1}=0.1$ )

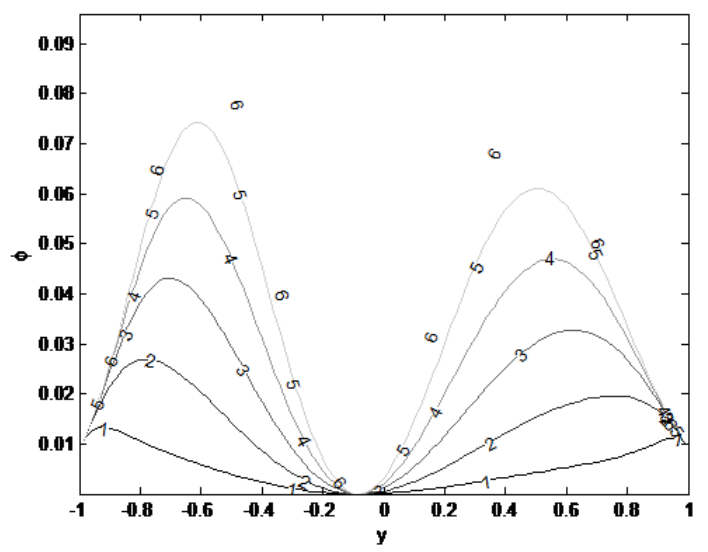

Fig. 7: Irreversibility ratio for different values of $\mathrm{Pe}(s=0.5, x=$ $0.1, p r=0.71, B r \Omega^{-1}=0.1$ )

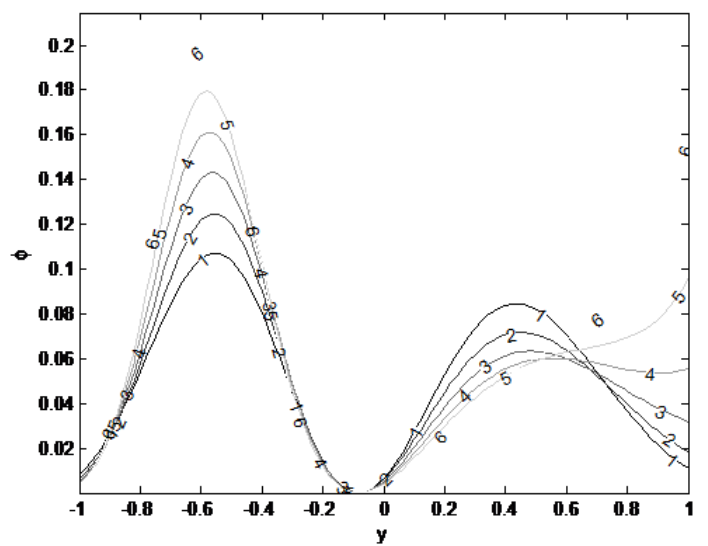

Fig. 8: Irreversibility ratio for different values of $\operatorname{Pr}(s=0.5, x=$ $0.1, P e=7.1, B r \Omega^{-1}=0.1$ )

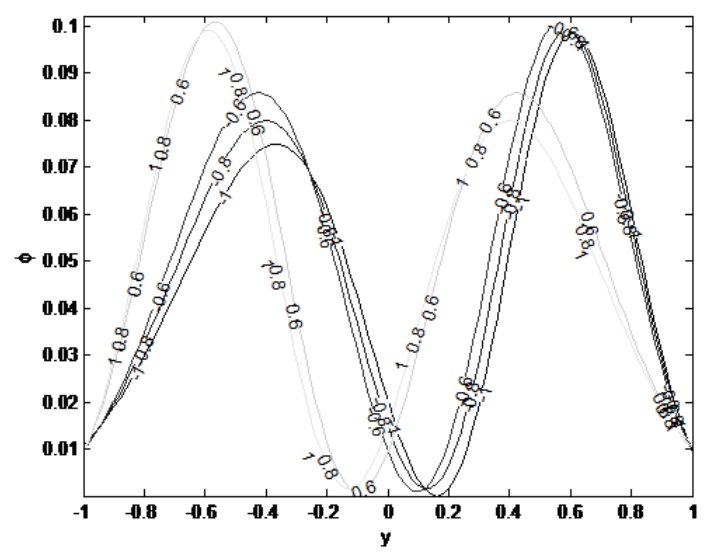

Fig. 9: Irreversibility ratio for different values of $\mathrm{s}(x=0.1, p r=$ $0.71, p e=7.1, \mathrm{Br}^{-1}=0.1$ ) 


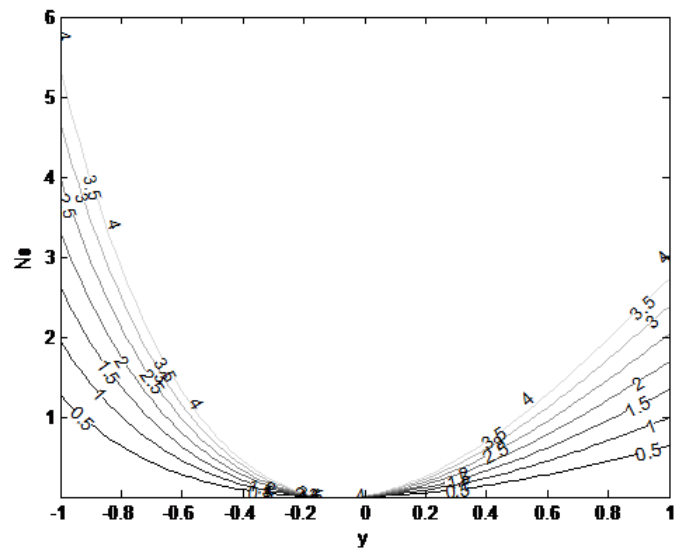

Fig. 10: Entropy generation for different values of $B r \Omega^{-1}(x=$ $0.5, \operatorname{Pr}=0.71, P e=7.1, s=0.5$ )

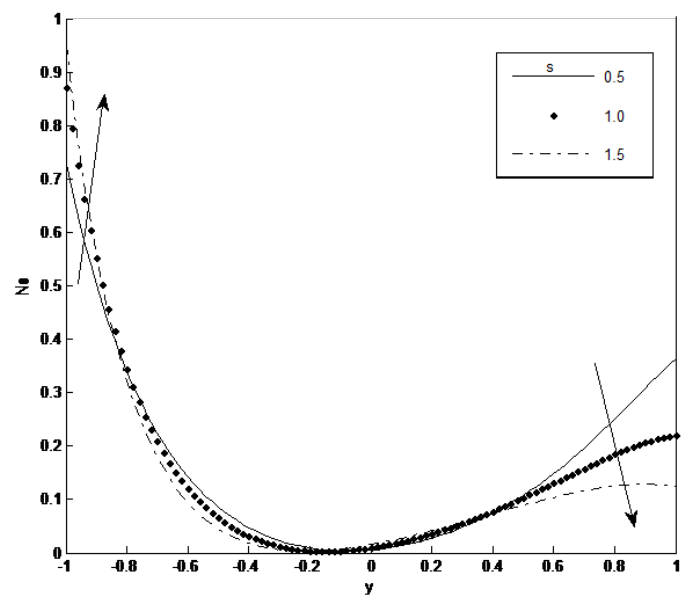

Fig. 11: Entropy generation for different values of $s(x=0.1, P r=$ $0.71, \mathrm{Pe}=7.1, \mathrm{Br} \Omega^{-1}=0.1$ )

perature profile are obtained and used to compute the entropy generation and irreversibility ratio for different values of suction/injection parameter $(s)$, Prandtl number $(\mathrm{Pr})$, group parameter $\left(\mathrm{Br} \Omega^{-1}\right)$ and Peclet number $(\mathrm{Pe})$. Generally, we observed that;

1. Entropy generation on the suction wall is higher when compared to the entropy on the wall with injection, this indicates that suction of fluid on the system encourages entropy generation.

2. Entropy generation near the two porous walls is higher than entropy generation within the channel, indicating that friction due to surface of the two walls increases entropy.

3. Entropy generation due to heat transfer dominates the irreversibility for different values of dimensionless suction/injection parameter (s), Prandtl number (Pr), and Peclet number $(\mathrm{Pe})$.

4. Entropy generation due to viscous dissipation as a result of fluid friction dominates the irreversibility ratio for values of group parameter $\left(B r \Omega^{-1} \geq 1\right)$.

\section{List of Symbols}

\begin{tabular}{|c|c|}
\hline$B e$ & Bejan number \\
\hline$B r$ & Brinkman number \\
\hline$E_{C}$ & Eckert number \\
\hline$E_{G}$ & volumetric rate of entropy generation \\
\hline$E_{G, C}$ & $\begin{array}{l}\text { characteristic entropy transfer rate } g \text { acceleration } \\
\text { due to gravity }\end{array}$ \\
\hline$h$ & channel width, half width of the channel \\
\hline$N_{x}$ & $\begin{array}{l}\text { entropy generation by heat transfer due to axial } \\
\text { conduction }\end{array}$ \\
\hline$N_{f}$ & entropy generation by viscous dissipation \\
\hline$N_{S}$ & dimensionless entropy generation number \\
\hline$N_{y}$ & $\begin{array}{l}\text { entropy generation due to heat transfer across fluid } \\
\text { sections }\end{array}$ \\
\hline$P e$ & Peclet number \\
\hline $\operatorname{Pr}$ & Prandtl number \\
\hline$C p$ & specific heat at constant pressure \\
\hline$s$ & dimensionless suction/injection parameter \\
\hline$T_{0}$ & temperature at $y^{\prime}=0$ \\
\hline$T^{\prime}$ & dimensional fluid temperature \\
\hline$T_{w}$ & temperature of plate at $y^{\prime}= \pm h$ \\
\hline$T$ & dimensionless temperature of fluid \\
\hline$u^{\prime}$ & dimensional velocity of fluid \\
\hline$U$ & $\begin{array}{l}\text { dimensionless velocity of fluid } \mathrm{U} \text { dimensional ve- } \\
\text { locity of the moving plate }\end{array}$ \\
\hline$\vec{V}$ & $\begin{array}{l}\text { velocity vector having components } u, v \text {, win the } \\
\text { directions } i, j, k\end{array}$ \\
\hline$v_{0}$ & velocity of suction/injection \\
\hline$x^{\prime}$ & horizontal axis (direction of flow) \\
\hline$x$ & dimensionless horizontal axis \\
\hline$y^{\prime}$ & co-ordinate perpendicular to the plate \\
\hline$y$ & dimensionless vertical co-ordinate \\
\hline$\theta$ & angle of inclination \\
\hline
\end{tabular}

\section{Greek alphabets}

$\begin{array}{ll}\alpha & \text { thermal diffusivity } \\ k & \text { thermal conductivity } \\ \phi & \text { irreversibility distribution ratio } \\ \mu & \text { coefficient of viscosity } \\ \rho & \text { density of the fluid } \\ \Omega & \text { dimensionless temperature difference }\end{array}$




\section{References}

[1] Ramana Murthy J.V., Srinivas J., Second Law Analysis for Poiseuille flow of immiscible micropolar fluids in a channel, Int. J. Heat Transfer., 2013,65, 0 254-264.

[2] Bejan A., Second law analysis in heat transfer, Energy Int. J., 1980, 5, 0 721-732.

[3] Bejan A., Entropy Generation through Heat and Fluid Flow, 1994, Chapter 5, 98, Wiley, Canada.

[4] Bejan A., Entropy Generation Minimization, 1996, CRC Press, Boca Raton, FL, New York.

[5] Makinde O.D., Maserumule R.L., Thermal criticality and entropy analysis for a variable viscosity Couette flow, Phys. Scr., 2008, 78, 0 1-6.

[6] Makinde O.D., Gbolagade A.W., Second law analysis of incompressible viscous flow through an inclined channel with isothermal walls, Rom. Journ. Phys., 2005, 50, 923-930.

[7] Makinde O.D., Osalusi E., Entropy generation in a liquid film falling along an incline porous heated plate, Mech. Res. Comm., 2006, 33, 692-698.

[8] Ajibade A.O., Jha B.K., Omame A., Entropy generation under the effect of suction/injection, Appl. Math. Model., 2011, 35 , 4630-4646.

[9] Hooman K., Hooman F., Mohebpour S.R., Entropy generation for forced convection in a porous channel with isoflux or isothermal walls, Int. J. Exergy, 2008, 5(1), 78-96.

[10] Aziz A., Entropy generation in pressure gradient assisted Couette flow with different thermal boundary conditions, Entropy, 2006, 8(2), 50-62.

[11] Makinde O.D., Second law analysis for variable viscosity hydromagnetic boundary layer flow with thermal radiation and Newtonian heating, Entropy, 2011, 13, 1446-1464.
[12] Makinde O.D., Aziz A., Second law analysis for a variable viscosity plane Poiseuille flow with asymmetric convective cooling, Comp. Math. Appl., 2010, 60(11), 3012-3019.

[13] Vyas P., Ranjan A., Entropy analysis for MHD generalized Couette flow in a composite Duct, journal of industrial mathematics, Entropy, 2006, 8(2), 50-62.

[14] Mahian O., Oztop H.F., Pop I., Mahmud S., Wongwises S., Design of a vertical annulus with MHD flow using entropy generation analysis, thermal science, 17(4) (2013) 1013-1022.

[15] Mahian O., Pop I., Sahin A.Z., Wongwises S., Estimation of operational costs due to entropy generation in a vertical annulus, Int. J. Exergy, 2013, 13(4), 472-486.

[16] Makinde O.D., Thermal analysis of a reactive generalized Couette flow of power law fluids between concentric cylindrical pipes, Europ. Phys. J. Plus, 2014, 129(270), 1-9.

[17] Bejan A., A study of entropy generation in fundamental convective heat transfer, Trans. ASME J. Heat Transf., 1979, 101, 718-725.

[18] White F.M., Viscous Fluid flow, 1974, McGraw-Hill, New York.

[19] Bejan A., Second law analysis in heat transfer and thermal design, Adv. Heat Transf., 1982, 15, 1-58.

[20] Paoletti S., Rispoli F., Sciubba E., Calculation of exegetic losses in compact heat exchanger passages, ASME AES, 1989, 10, 21-29.

[21] Bejan A., Convection heat transfer, 1984, Hoboken, Wiley.

[22] Hooman K., Gurgenci H., Effects of temperature-dependent viscosity variation on entropy generation, heat and fluid flow through a porous-saturated duct of rectangular cross-section, Appl. Math. Mech., 2007, 28(1), 69-78.

[23] Bejan A., A Study of Entropy Generation in Fundamental Convective Heat Transfer, J. Heat Transf., 1979, 101(4), 718-725.

[24] Lienhard IV J.H., Lienhard V J.H., A Heat Transfer Textbook (version 1.24), 2006, 707-718, 3rd ed., Phlogiston Press, Cambridge, MA. 